\title{
全球-地方出口溢出效应对新企业 进入出口市场的影响
}

\author{
贺灿飞 ${ }^{1,2}$, 胡绪千 ${ }^{1,2}$, 罗 芉 ${ }^{1,2}$
}

(1. 北京大学城市与环境学院, 北京 100871 ;

2. 北京大学-林肯研究院城市发展与土地政策研究中心, 北京 100871)

\begin{abstract}
摘 要: 出口增长是驱动中国经济发展的关键力量, 新出口企业进人是出口增长的微观体现。新贸易理论认为集聚 外部性带来的知识溢出是影响企业出口决策的关键因素。出口地、目的国维度的知识溢出均有利于企业获取必要 的出口知识, 降低进入出口市场的难度。演化经济地理理论强调认知邻近是出口溢出发挥作用的前提, 出口地、目 的国维度的出口经验、产品关联是出口溢出的主要内容。论文利用 2002-2011年中国海关贸易数据库, 分析出口 地、目的国溢出对新企业进入出口市场的作用。结果发现: (1) 出口溢出可以显著提升新出口企业进入概率, 私营 企业更倾向于进人出口溢出更强的出口地、目的国市场; (2) 出口溢出对跟随型企业, 特别是本土跟随型企业进人 的促进作用更强, 国有集体企业更强调整体出口经验溢出, 私营企业更强调产品知识溢出; (3) 出口地溢出有利于 新出口企业拓展到新目的国, 目的国溢出有利于新出口企业拓展到新产品。论文完善了出口企业空间动态研究, 拓展了出口溢出对出口决策作用的空间维度, 也有助于深化对中国贸易空间的理解。
\end{abstract}

关 键 词: 出口溢出; 全球-地方互动; 新出口企业进人; 中国

贸易是驱动中国经济增长的重要力量。改革 开放以来,伴随着全球化不断推进, 中国对外贸易 迅速发展。企业是贸易活动的微观主体。一国贸 易增长的背后存在着大量企业出口关系的建立和 终止、新企业的进人与退出等动态行为。Bernard 等(2004)研究发现美国 70\%的贸易增长是由进人出 口市场的新企业带来的。因此, 研究企业出口动 态, 特别是新出口企业的空间进人动态有利于解读 对外贸易空间, 更深刻地理解中国经济地理格局的 时空演变。

国际贸易理论为出口行为的产生机制及其空 间分布规律提供了解释。新贸易理论强调在集聚 外部性作用下, 出口企业为获取本地知识溢出和技 术扩散,进而实现规模报酬递增, 有在空间上相互
邻近的集聚动力 (Krugman, 1979; Ottaviano et al, 2002)。新新贸易理论细化到微观的企业层面, 指 出企业必须支付固定的沉没成本, 才能顺利进入出 口市场, 而且企业出口决策会由于各自属性特征的 不同而存在差异 (Melitz, 2003; Baldwin et al, 2007)。有关企业异质性的研究多将生产率作为区 分企业异质性的关键指标。也有部分学者将产品 质量、企业规模等因素纳人研究,但针对其他异质 性的探讨相对较少(Baldwin et al, 2007)。

对于本身无出口经验的新出口企业来说, 学习 外界出口知识对其跨越出口市场进人门槛至关重 要。在地方维度, 出口企业之间空间邻近有利于出 口信息的共享交流,使潜在出口企业更容易克服沉 没成本, 开始对外出口 (Grabher, 2002; 郭琪,

收稿日期: 2018-09-05; 修订日期: 2019-02-03。

基金项目: 国家杰出青年科学基金项目(41425001); 国家自然科学基金重点项目(41731278)。[Foundation: National Natural Science Funds for Distinguished Young Scholar, No. 41425001; Major Program of National Natural Science Foundation of China, No. 41731278. ]

第一作者简介: 贺灿飞(1972- ), 男,江西永新人,教授,博士生导师,主要从事经济地理、产业与区域经济研究。

E-mail: hecanfei@urban.pku.edu.cn

引用格式: 贺灿飞, 胡绪千, 罗芉. 2019. 全球-地方出口溢出效应对新企业进入出口市场的影响 [J]. 地理科学进展, 38(5): 731-744. [He C F, Hu X Q, Luo Q. 2019. Impact of export spillovers on the entry of new firms into the export market. Progress in Geography, 38(5): 731744. ] DOI: $10.18306 /$ dlkxjz.2019.05.010 
2016)。较多学者这一视角出发, 验证了出口集聚 对出口决策的正向作用(Aitken et al, 1997; Malmberg et al, 2000; Koeing, 2009)。除地方尺度外, 区 域与外界联系导人的知识和信息同样可以成为企 业学习的来源。贸易活动将全球范围内的经济活 动主体链接起来, 并借助进出口关系推动知识流动 (Rinallo et al, 2010)。因此, 新出口企业不仅要关注 集群内知识溢出强度, 也要关注集群对外贸易联系 是否可以带来更多为自己所用的外部知识。但是, 目前有关知识溢出与企业出口决策的研究, 或从出 口地维度出发, 或从目的国维度出发, 较少将 2 个维 度结合起来, 分析新企业进人出口市场与全球一地 方出口溢出的关系。

演化经济地理理论为知识溢出的前提条件和 具体内容给出了解释。在集聚外部性作用下, 与所 在集群存在适当“认知邻近性”(cognitive proximity) 的新企业能够以更高的效率整合集群内知识, 实现 对外出口(Boschma et al, 2007)。因此, 新出口企业 在空间上出现的概率取决于与其具备特定认知邻 近性的学习来源所在区位。另一方面, 出口地与目 的国维度溢出的出口经验是新出口企业需要的重 要贸易 “能力”, 是新出口企业重要的学习来源。如 果潜在出口企业计划出口的产品与出口地或目的 国已出口产品集合的技术关联较高, 那么 2 种产品 在生产技术、生产设备、研发能力、劳动力和目标市 场等方面可以分享的知识容量也越大。而遵循既 有的产品出口经验, 选择进人与潜在出口产品技术 关联较大的区域作为出口地或目的国, 同样可以利 用关联产品间知识的相似性来降低新出口企业用 于研发技术、改善产品的成本, 使之进人出口市场 的难度降低(Dosi, 1997)。综上, 本文一方面基于现 实背景, 研究中国出口市场新企业进人的格局和机 制, 深化对中国出口空间的认知; 另一方面, 基于国 际贸易、演化经济地理和知识溢出等理论前沿, 研 究全球、地方出口溢出对不同类型新出口企业的作 用, 以完善出口企业空间动态研究, 拓展出口溢出 对出口决策作用的空间维度, 加深新新贸易理论对 企业异质性的理解。

\section{1 理论框架}

对于本身无出口经验的新出口企业而言, 其所 在集群的产品知识和出口信息对跨越出口市场门
槛, 实现出口额从零到有的突破至关重要。由于出 口行为与一般生产行为不同, 涉及出口地-目的国 2 个空间维度, 因此有必要跳出地方视角, 在讨论地 方集聚外部性如何提升新出口企业进人可能性的 基础上, 同时关注地方通过与全球构建联系引人的 外部知识在吸引新企业进人方面会赋予城市怎样 的影响。结合新贸易理论强调的“集聚外部性”和 演化经济地理学提出的“认知邻近性”, 本文从地方 和全球 2 个维度提出研究理论框架。

\section{1 集聚外部性与新出口企业进入}

集聚经济是经济活动在空间上相互邻近带来 的成本节约和效益提升(韦伯, 1997)。知识溢出是 集聚和集群形成并能够通过自我强化实现规模扩 张的驱动力。Marshall(1920)在早期曾经提出 “产业 氛围” (industrial atmosphere)的概念, 用以形象化地 描述集聚可能带来的外部性影响。对于出口企业 而言, 其获取知识溢出的途径不再局限于集群内 部, 集群与外界联系导人的知识和信息同样可以成 为企业学习的来源。

\subsection{1 城市出口经验}

一方面, 出口企业可以受益于地方集聚。 Bathelt 等(2004)提出 “地方蜂鸣” (Local Buzz)的概 念, 形象地描述了这一作用, 即企业选址于特定集 群这一区位行为本身就会使其受益于集群内部的 信息扩散、知识学习和交流共享(Gertler, 1995)。这 种知识溢出的存在也令企业能够更容易地获取资 源、评估风险、面对未知市场。同区域已有出口企 业集聚可以激发出口经验溢出, 从而赋予潜在出口 企业巨大的信息优势,使之更方便地接触到成功的 出口经验, 降低搜寻出口信息的沉没成本,降低进 人出口市场的难度。此外, 区域出口经验越丰富, 对外贸易传统越深厚, 各区域主体越有动机主动调 整制度, 创造对出口行为更加友好包容的集群氛 围, 为新出口企业提供发展机会 (Maskell et al, 2007; Martin, 2010)。

\subsection{2 目的国出口经验}

另一方面, 出口企业也受到全球联系的影响。 企业从某出口地到目的国的出口行为不仅仅与本 身相关，更牵涉着政府、消费者、中间商等利益主 体, 它们共同构成了全球尺度的生产网络。贸易活 动将全球范围内的区域和经济活动主体链接起来, 并且借助进出口关系推动二者间的知识流动(Rinallo et al, 2010)。在这一过程中, 地方企业可以获得 
本身不具备的外来知识, Bathelt等(2010)将这些外 来知识称为 “全球蜂鸣”(Global Buzz)。贸易行为在 生产网络中的实现,推动产品技术、目的国制度、市 场偏好等知识得以超越地理距离传递(Bathelt et al, 2010)。目的国出口经验溢出对潜在出口企业的出 口决策具有指导作用(Rinallo et al, 2010)。如果潜 在出口企业能够借助区域对外贸易联系获取到丰 富的目的国出口经验,那么该企业就可以借助其中 隐含的产品、市场、制度信息, 掌握更多有助于向该 目的国出口所需的知识技能, 提升出口成功概率。

\section{2 认知邻近性与新出口企业进入}

集聚外部性强调了地理邻近性无论是地方尺 度的集群内部, 抑或是全球尺度的集群对外贸易网 络都能为潜在的出口企业提供出口经验。而演化 经济地理学则进一步提出, 与所在集群存在适当 “认知邻近性”的新企业能够以更高的效率地整合 集群内的编码知识和隐性知识, 从而有更大的概率 发展出竞争优势实现出口额从无到有的突破(Marshall, 1920; Jacobs, 1969; Boschma et al, 2007)。对 于潜在出口企业及其可获得的知识来说, 认知距离 太远不利于快速学习和转化, 而认知距离太近又容 易导致路径锁定, 只有认知距离处于合适的水平, 新出口企业才能实现有效学习 (Nooteboom, 2001)。为了量化 “认知距离”, Hidalgo 等(2007)和 Boschma 等 (2009) 利用 “技术关联” (technological relatedness) 衡量 “认知邻近性”, 并证实了地区内部 较强的技术关联对知识溢出发生的促进作用, 指出 技术关联是集聚效应产生的重要来源, 也是新技 术、新企业、新集群产生的重要推动力 (Boschma, 2005; Timmermans et al, 2014)。

\subsection{1 城市产品技术关联}

对于出口企业来说, 出口产品所包含的技术是 地方知识的重要承载。特定产品出口经验的溢出 会直接影响到潜在出口企业在产品方面的沉没成 本和创新风险。而且潜在出口企业计划出口的产 品与区域内已出口产品之间的认知邻近性越高, 新 出口企业需要主动搜寻的产品技术和知识越少, 在 产品研发、市场拓展等方面付出的代价也越小, 对 应出口行为的成功率便会相应上升(Boschma et al, 2012)。Poncet(2013)利用技术关联作为解释变量, 发现技术关联越大的产品对应的出口增长速度越 快。贺灿飞等(2016)以中国对外出口产品为研究对 象, 得出同样的结论: 在技术关联的作用下, 中国出
口产品演化呈现出路径依赖的特征。

\subsection{2 目的国产品技术关联}

目的国溢出的地理距离较远, 而且与出口地在 制度环境上差异较大。这使潜在出口企业在出口 决策时面临更大的不确定性。为此, 潜在出口企业 在学习目的国知识溢出时会采取一定策略, 吸取与 自身在组织、关系、技术等方面存在认知邻近的目 的国知识(Malecki, 2010)。因此, 在全球尺度的出 口益出过程中, 新出口企业学习目的国溢出的效果 更强调出口地与目的国之间是否能够在组织管理、 产品技术、消费者偏好等方面保有共识(Boschma, 2005; Torre, 2008)。本文认为新出口企业计划向特 定目的国出口的产品与中国出口到该目的国已有 出口产品空间之间的技术关联是刻画认知距离的 变量之一。目的国出口产品关联越高, 潜在出口企 业计划出口的产品与中国出口到目的国的已有产 品共享越多知识和信息, 越有利于新出口企业通过 解读目的国知识溢出提升向对应目的国出口对应 产品的概率。

\section{3 企业异质性与溢出效果差异}

新新贸易理论成功将贸易研究的对象细化到 微观的企业层面,关注企业出口决策问题。企业生 产率在 Melitz(2003)的研究中被作为区分企业能否 顺利进人出口市场的重要衡量指标, 因而也成为后 来新新贸易理论中用以界定企业异质性的关键变 量。本文试图对现有企业异质性的量化指标进行 补充。

一方面,所有制是企业的固有属性。不同所有 制企业的注册资本构成存在结构性差异, 学习知识 的能力也不同。外资企业有更先进的生产技术和 管理经验,在对接国外市场方面也保有先天优势。 国有集体企业受国家指令作用更大,其出口决策未 必遵循城市既有出口路径。私营企业是市场化改 革的产物, 其出口活动受成本最低和利润最高驱动 更明显, 其出口决策更看重地方生产禀赋和成本优 势(郭琪, 2016)。

除所有制外,进人类型不同的企业受出口溢出 影响也不同。本文按照是否进人新目的国、是否出 口新产品, 将新出口企业划分为 4 种。对于未出口 新产品也未进人新目的国的“跟随型企业”来说, 出 口地、目的国的成熟出口经验可能降低潜在出口企 业面临的成本和风险,催生企业作出出口决策(Boschma et al, 2012)。而对于出口新产品或出口到新 
目的国或出口新产品到新目的国的“突破型企业” 来说, 出口地、目的国溢出的信息是其整合形成新 资源, 进而实现出口路径突破, 向认知邻近的新产 品和新出口节点跳跃的基础(Chaney, 2014)。此时, 城市既有的产品关联、区域出口经验和出口目的地 溢出都可能为新出口企业跳跃到认知邻近的新产 品和新出口节点提供便利, 使之更容易发展出向新 目的国出口新产品的比较优势。

基于对理论基础、实证进展的综述, 以及对出 口地、目的国溢出对中国对外出口市场新企业进人 作用的分析,构建研究框架如图 1 所示。

\section{2 数据与方法}

\section{1 数据来源与核心变量度量}

核心变量包括新出口企业进人、出口地与目的 国的出口经验、产品技术关联等指标。对应的原始 数据来源均为中国海关贸易数据库。基于对数据 可得性和完整性考虑, 本文选取中国加人世界贸易 组织(WTO)后的 2002-2011 年即时间跨度为 $10 \mathrm{a}$ 的数据作为样本 ${ }^{\mathbb{1}}$ 。此外本文还对数据作了 2 个方 面的处理。其一, 贸易产品 HS 编码是国际贸易相 关方面共用的商品分类体系。在本文的研究时段 内, HS 编码体系经历过 2 次修订, 部分产品对应 HS 分类和编码发生变动。为保证产品前后分类一致， 根据联合国官网提供的编码调整文件 ${ }^{2}$, 将海关数 据库中的产品 HS 编码统一调整为 2007 年版本。其 二, 中国海关库中包含很多贸易公司, 而贸易公司 本身并不进行生产, 主要是作为帮助其他生产企业 完成出口报关等工作, 因此可能出现一家贸易公司 出口上百种产品的情况。在衡量影响出口产品的 决策时, 这类企业的产品跟普通生产厂商存在较大 差异, 因此本文剔除了数据中的贸易公司。剔除标 准主要是基于 Ahn 等(2011)与 Manova等(2012)的方 法, 剔除企业名称中含有 “贸易” “进出口” “出口” “进口”等字眼的企业, 此外, 还剔除了一些企业名 称中包含 “外贸” “商贸” “边贸” “物流” 等明显也不 从事产品生产的企业。

\subsection{1 新出口企业识别}

本文利用海关数据库中企业编码的唯一识别

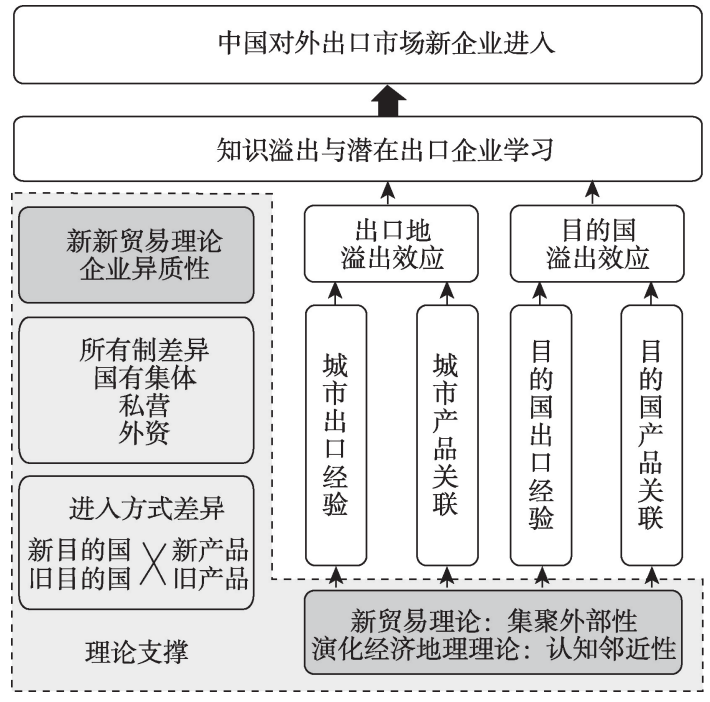

图 1 研究框架

Fig.1 Research framework

性, 识别进人出口市场的企业。如果特定编码出口 企业在 $t$ 年不存在于数据库中, 而 $(t+1)$ 年存在, 则将 该企业识别为 $t$ 年进人出口市场的新企业。计算 $t$ 年进人出口市场的企业数与已有出口企业数的比 值, 即为企业进人率:

$$
\mathrm{ER}_{t}=\frac{E_{t}}{\operatorname{Exp}_{t}}
$$

式中: $\mathrm{ER}_{t}$ 为新出口企业进入率; $E_{t}$ 为新出口企业 进人数; $\operatorname{Exp}_{t}$ 为出口企业总数。

为防止出现已有出口企业在新出口地开设分 支机构,或者已有出口企业退出某地出口市场的同 时转而进人另一地出口市场, 却被识别为新出口企 业的情况(上述识别过程不涉及地理维度), 在识别 出新出口企业之后, 将新出口企业重新对应到出口 地和目的国层面,计算各出口地、目的国的新出口 企业进人率, 公式如下:

$$
\mathrm{ER}_{c s t}=\frac{E_{c s t}}{\operatorname{Exp}_{c s t}}
$$

式中: $\mathrm{ER}_{c s t}$ 为 $t$ 年 $c$ 城市向 $s$ 目的国新出口企业进人 率; $E_{c s t}$ 为 $t$ 年 $c$ 城市向 $s$ 目的国新出口企业进人数; $\operatorname{Exp}_{c s t}$ 为 $t$ 年 $c$ 城市向 $s$ 目的国出口企业总数。

\subsection{2 城市、目的国出口经验}

Koenig 等(2010)认为出口溢出效应的强度与已 有出口企业的规模无关,与集聚企业的数量有关。 本文据此计算 $t$ 年 $c$ 城市已有的出口企业数量 $\operatorname{Exp}_{c t}$

(1) 由于企业的进入(退出)的识别,需要连续 $2 \mathrm{a}$ 的信息,因此本文最终以 2002-2010年企业的进入(退出)情况作为样本分析。

(2) https://unstats.un.org/unsd/trade/classifications/correspondence-tables.asp。 
表征出口地出口经验; 计算 $t$ 年 $s$ 目的国已有的中国 出口企业数量 $\operatorname{Exp}_{s t}$ 表征目的国出口经验。

\subsection{3 城市、目的国产品技术关联}

本文模仿 Hidalgo 等(2007)提出的方法, 通过计 算产品共现概率的方法度量城市出口产品技术关 联。首先,计算任意 2 种产品被同一城市同时出口 的条件概率, 如果 2 种产品经常同时被一个城市出 口, 则这 2 种产品在技术、劳动力、资本等方面对城 市有相似的需求。

$$
\varnothing_{p_{1} p_{2}}=\min \left\{P\left(V_{c p_{1}}>0 \mid V_{c p_{2}}>0\right), P\left(V_{c p_{2}}>0 \mid V_{c p_{1}}>0\right)\right\}(3)
$$

式中: $c$ 代表城市; $p_{1} 、 p_{2}$ 代表 2 种 4 位 HS 编码产 品; $V$ 代表出口额。 $\varnothing_{p_{1} p_{2}}$ 是 2 种产品同时被同一城 市出口的条件概率最小值。 $\varnothing_{p_{1} p_{2}}$ 越大, 说明 2 种产 品共现概率越大, 技术关联越高。

之后, 计算特定产品与城市出口产品空间的技 术关联 $\operatorname{Den}_{c p}$, 公式如下:

$$
\operatorname{Den}_{c p}=\frac{\sum_{p_{i}} x_{c p_{i}} \varnothing_{p p_{i}}}{\sum_{p_{i}} \varnothing_{p p_{i}}}
$$

式中: $x_{c p_{i}}$ 代表城市 $c$ 的出口产品 $p_{i}$ 是否为优势产 品, 是则取值 1 , 否则取值 0 。优势产品通过计算区 位商衡量, 如果城市 $c$ 的出口产品 $p_{i}$ 的区位商 $\mathrm{LQ}_{c p_{i}}$ 大于 1 , 则将其看作优势产品, $\mathrm{LQ}_{c p_{i}}$ 计算公式如下:

$$
\mathrm{LQ}_{c p_{i}}=\frac{V_{c p_{i}} / \sum_{p_{i}} V_{c p_{i}}}{\sum_{c} V_{c p_{i}} / \sum_{c, p_{i}} V_{c p_{i}}}
$$

采取同样的方法计算中国出口到特定目的国 的产品技术关联 $\operatorname{Den}_{s p}$ 。

\section{2 模型设定}

在描述性分析的基础上, 为具体分析出口溢出 对新企业进人出口市场的影响, 本文选取 20022011 年作为研究时段, 构建年份一地级市一目的国 -4 位 HS 编码维度的回归模型, 分别探讨有无新出 口企业进人、新出口企业中有无跟随型企业进人、 突破型企业中有无突破到新目的国的企业进人与 出口地、目的国溢出的关系, 并在此基础上进一步 将样本按照所有制进行划分, 分析所有制差异是否 会导致出口溢出的作用呈现进一步分异。

Entry $_{c s p t}=\beta_{0}+\beta_{1}$ City_Exp ${ }_{c t}+\beta_{2}$ City_Den $_{c p t}+$

$$
\beta_{3} \text { Country_Exp }{ }_{s t}+\beta_{4} \text { Country_Den }_{s p t}+
$$$$
\beta_{5} \text { City_PGDP } c t+\beta_{6} \text { Dis }_{s}+\beta_{7} \text { Country_Pop }_{s t}+
$$$$
\beta_{8} \text { Country_Ins }_{s t}+\varepsilon_{c s p t}
$$

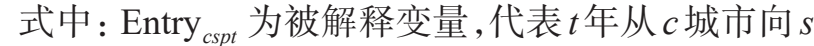
目的国出口 $p$ 产品的市场中是否有新企业进人。 City_Exp p $_{c t}$ 和 City_Den $c p t$ 分别为 $t$ 年 $c$ 城市的出口 经验和 $t$ 年 $c$ 城市 $p$ 产品的技术关联, 代表出口地维 度的知识溢出; Country_Exp s $_{s t}$ 和 Country_Den spt $_{\text {分 }}$ 别为 $t$ 年 $s$ 目的国的出口经验和 $t$ 年 $s$ 目的国 $p$ 产品 的技术关联,代表目的国维度的知识溢出。控制变 量中, City_PGDP $c t$ 为 $t$ 年 $c$ 城市的人均 GDP, 控制 出口地社会经济发展水平对新企业进人的影响, 数 据来源为中国区域经济统计年鉴; $\mathrm{Dis}_{s}$ 为中国与目 的国最大城市间距离,控制中国与目的国间地理距 离对出口溢出效果的影响, 数据来源为 CEPII 数据 库; Country_Pop st $_{\text {st }}$ 目的国人口数量, 控制目的国 市场规模的影响, 数据来源为 CEPII 数据库; Country_Ins st $_{\text {st }}$ 目的国社会稳定程度, 控制目的国 制度环境对新企业进人的影响, 数据来源为世界银 行治理数据库(WGI Dataset)。

\section{3 中国出口市场中新企业进人的空间 格局}

图 2 为对外出口市场新企业进人的空间格局及

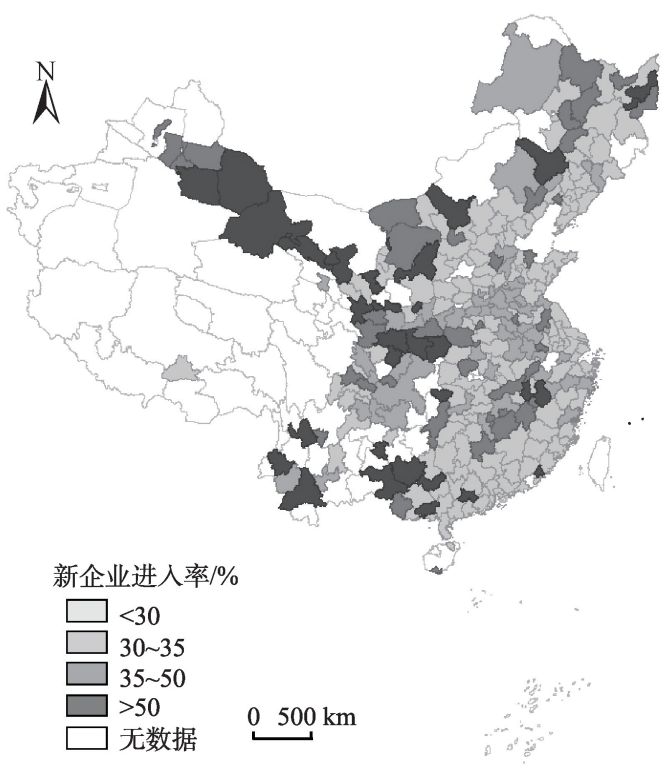

注:本图基于国家测绘地理信息局标准地图服务网站下载的 审图号为 GS(2016)2888 号的标准地图制作, 底图无修改。下同。

图2 2002-2010年出口地维度新出口企业进人空间格局

Fig.2 Spatial pattern of new export firms' entry in the export place dimension, 2002-2010 
其与出口地、目的国溢出的相关性分析结果。首先 在地级市层面统计 2002-2010 年新出口企业年均 进人率。沿海地区贸易规模大, 已有出口企业数量 多, 新出口企业所占比重较小。而伴随着中国对外 开放向内陆拓展, 中西部出口市场迅速扩张, 出口 企业动态活跃, 进入率较高。然后, 观察跟随型企 业(未进人新目的国且未进人新产品)和突破型企业 (进人新目的国或进入新产品)所占比重的空间格局 发现, 突破型企业主要分布在中西部地区(图3a), 而 跟随型企业主要分布在东部沿海地区(图 3b)。跟随 型企业选择城市既有的出口产品和目的国, 表现为 对已有出口路径的遵循。沿海地区出口产品多样, 目的国众多, 贸易规模庞大, 延续已有路径可以充 分利用出口经验, 降低沉没成本。而突破型企业实 现了出口路径创造。中西部地区全球化程度不高, 出口经验薄弱, 选择既有出口路径可节约的成本可 能不足以弥补与已有出口企业竞争需要付出的代 价。这时企业可能为了获取先发优势而选择新产 品以及新目的国。

从目的国维度进一步分析新出口企业地理格 局。图 4 展示了 2002-2010 年新出口企业年均进 人率, 部分进人率较高的国家与中国在地理上相互 邻近, 如韩国、越南、蒙古、印度等; 另一部分进人率 较高的国家是中国的主要贸易伙伴, 如美国、澳大 利亚等。图 5 反映了目的国维度突破型、跟随型新
出口企业数量比重的空间分布。跟随型企业所占 比重较高的经济体包括日本、韩国、美国、澳大利 亚、英国、法国等。这些国家与中国已有的贸易联 系较强, 不仅会引导新出口企业遵循已有的出口路 径以降低学习成本, 提升出口可能性, 而且其本身 留给新出口企业实现路径创造的空间相对较小。 与跟随型企业的数量比重分布相反, 突破型企业占 比较高的经济体主要分布于非洲、大洋洲。这些区 域与中国的贸易联系薄弱, 出口产品和目的国也相 对单一。因此, 新出口企业延续历史出口路径可获 取的信息较少, 且一旦有新出口企业进人,便极有 可能实现新目的国和新产品的突破。

首先, 明确新企业进人与城市出口经验的相关 性。以某年某城市进人出口市场的新企业数量为 纵坐标, 该年该城市出口企业总数为横坐标, 绘制 散点图, 并添加线性趋势线, 得到图 $6 \mathrm{a}$ 。线性趋势 线的斜率为正, 表明城市出口经验与新出口企业出 现存在正相关关系, 丰富的城市出口经验可能有利 于新出口企业进人出口市场。这与前文分析相符, 城市内出口企业集聚引发的知识溢出使潜在出口 企业可以通过面对面交流、学习和模仿等方式掌握 出口经验, 提升了新出口企业出口行为在当地出现 的可能性。接下来分析城市产品技术关联对出口 市场新企业进人的作用。图 6b 对比了 2002-2010 年间有无新企业进人的城市-产品组合对应产品技 (a) 突破型企业

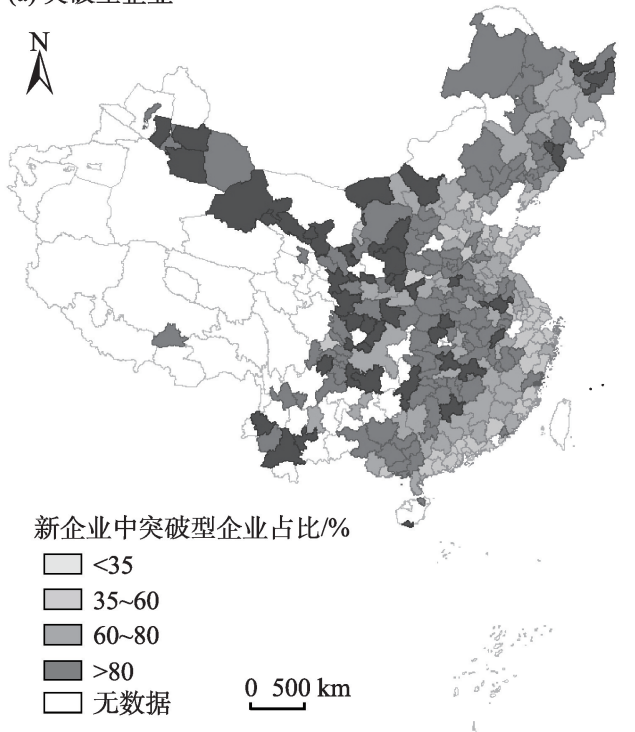

(b) 跟随型企业

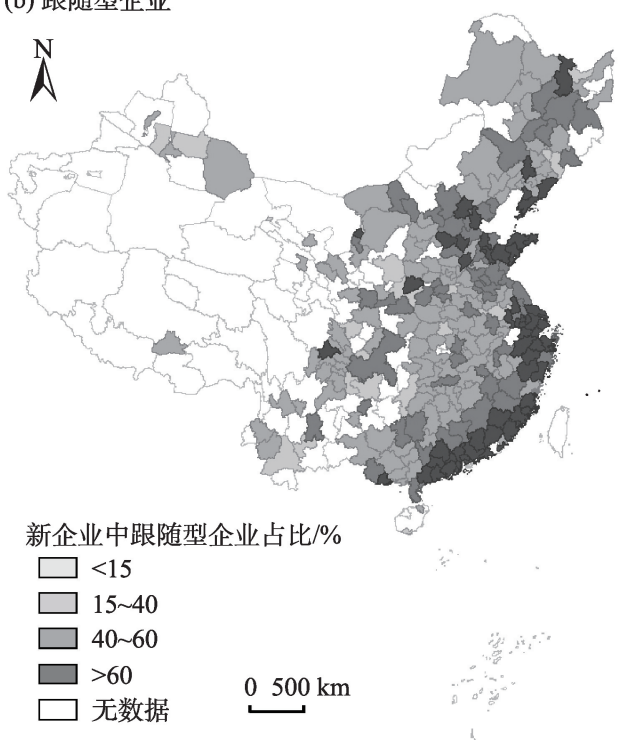

图 3 2002-2010年出口地维度新出口企业中突破型企业和跟随型企业所占比重的空间格局

Fig.3 Spatial pattern of the proportion of breakthrough and follower firms in new exporters in the export place dimension, 2002-2010 
术关联的概率密度分布。可以发现, 存在新出口企 业进人的城市-产品组合的概率密度分布曲线更靠 右, 表明新出口产品与当地已出口的技术关联程度
更高。这在一定程度预示了潜在出口企业计划出 口的产品与城市出口产品空间邻近度越高, 二者在 产品研发生产等方面共享的知识越丰富, 产品出口

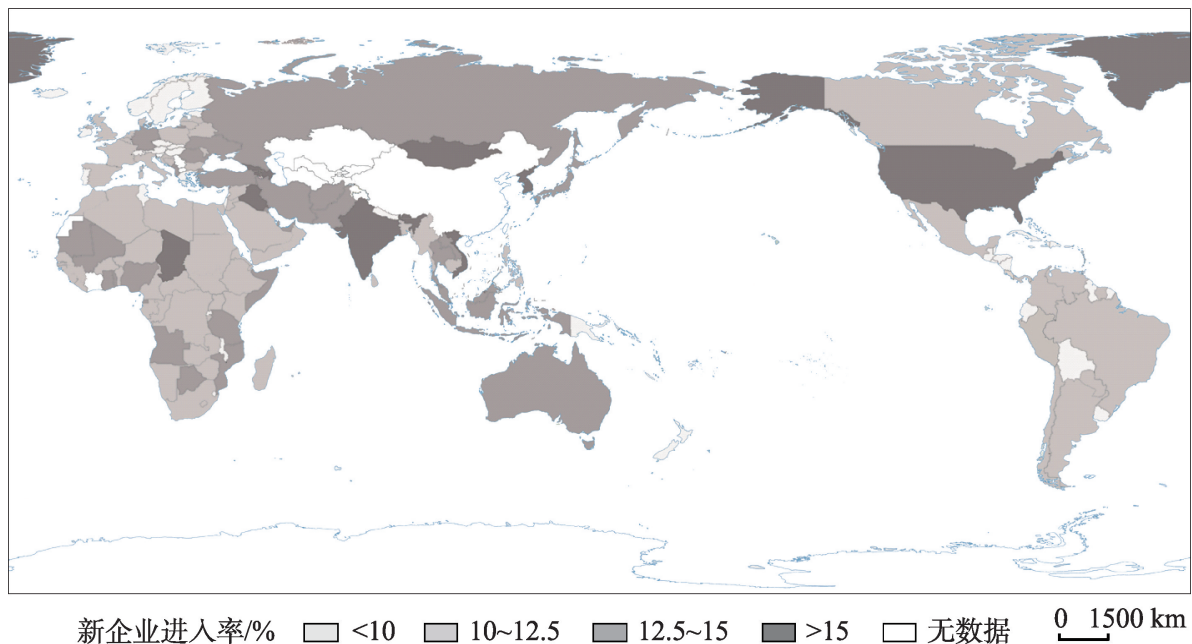

注: 本图基于国家测绘地理信息局标准地图服务网站下载的审图号为 GS(2016) 1663 号的标准地图制作,底图无修改。下同。

图4 2002-2010年目的国维度新出口企业进人空间格局

Fig.4 Spatial pattern of new export firms' entry in the export destination dimension, 2002-2010

(a) 突破型企业

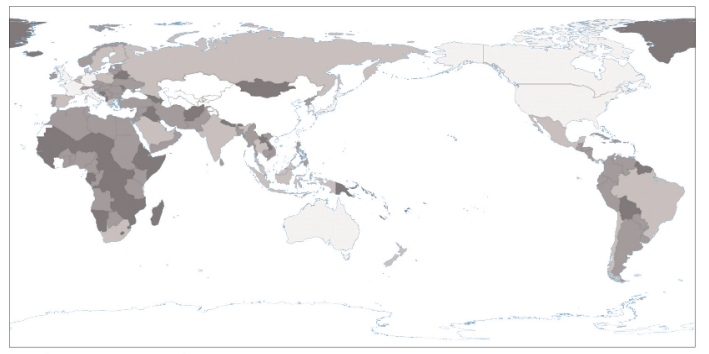

新企业进人率 $/ \%$

$\square<10 \square 10 \sim 12.5 \square 12.5 \sim 15 \square>15$ 口无数据 $01500 \mathrm{~km}$ (b) 跟随型企业

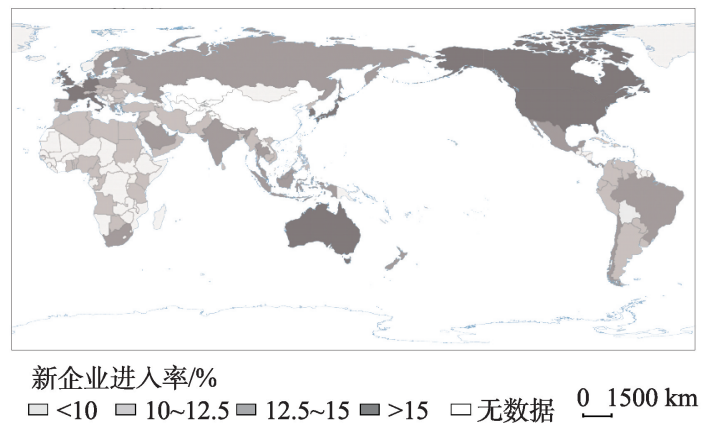

图 5 2002-2010年目的国维度新出口企业中突破型企业和跟随型企业所占比重的空间格局

Fig.5 Spatial pattern of the proportion of breakthrough and follower firms in new exporters in the export destination dimension, 2002-2010
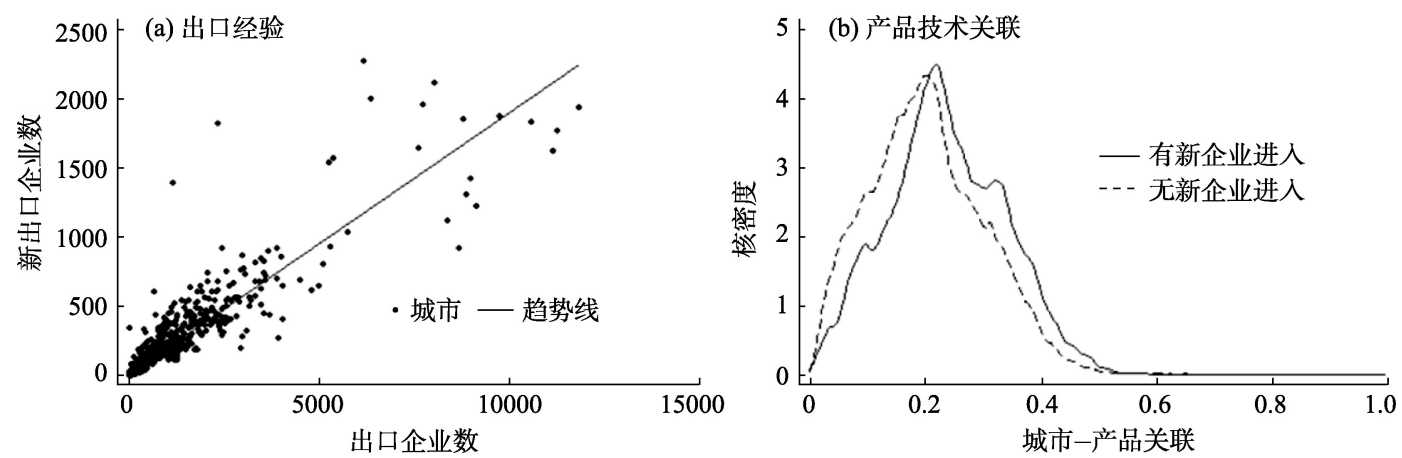

图 6 新企业进人与出口地溢出

Fig.6 New export firms' entry and spillover of export place 
经验对潜在出口企业的溢出效果越明显。

其次, 分析目的国知识溢出对新出口企业进人 的相关性。图 7a 展示了新出口企业进人与目的国 出口经验之间的关系, 发现目的国出口经验与新出 口企业出现的概率呈明显的正相关关系。这在一 定程度上预示目的国保有的以中国为出口来源地 的企业经验越丰富, 新出口企业可学习到的目的国 知识和信息越多, 进入出口市场的可能性越高。接 下来分析目的国产品技术关联对出口市场新企业 进人的相关性。图 $7 \mathrm{~b}$ 对比了有无新企业进人的目 的国产品技术关联的核密度分布。可以发现存在 新的城市一产品组合的出口目的国的产品技术关联 更紧密, 这预示新出口企业更有可能进人产品技术 关联更高的目的国。当新出口企业的出口产品与 中国对目的国已有出口产品空间在认知上相互邻 近时, 新出口企业可能从目的国溢出的产品信息中 搜集到对提升自身产品出口能力更有价值的部分,
使之在获取目的国产品信息方面拥有其他潜在出 口企业不具备的优势, 因而进人出口市场的可能性 也更大。

\section{4 实证分析}

\section{1 有无新出口企业进入与出口溢出}

从城市一目的国一产品维度出发, 分析出口溢 出对新企业进人出口市场的作用, 所得回归结果如 表 1 所示。其中模型 1 不区分所有制。模型 2 至模 型 4 区分所有制, 识别潜在的国有集体企业、私营企 业以及外资企业的出口决策对不同类型知识溢出 的需求差异。

模型 1 中,城市、目的国层面的出口经验和产品 技术关联对应的回归系数均显著为正, 说明出口溢 出的确可以显著促进潜在出口企业进人。城市中 已有出口企业数量越多, 区域保有的出口经验
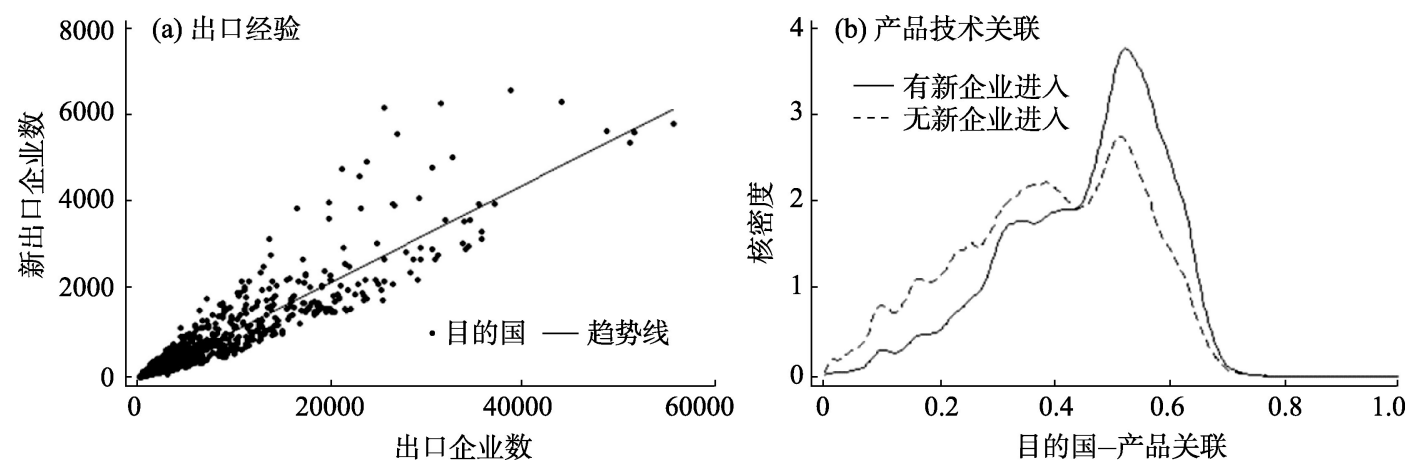

图 7 新企业进人与出口目的国溢出

Fig.7 New export firms' entry and spillover of export destination

表 1 有无新出口企业进入与出口溢出

Tab.1 New export firms' entry and export spillover

\begin{tabular}{|c|c|c|c|c|}
\hline \multirow{2}{*}{ 变量 } & 模型 1 & 模型 2 & 模型 3 & 模型4 \\
\hline & 不区分所有制 & 国有集体 & 私营 & 外资 \\
\hline City_Exp & $1.380^{* * * * *}$ & $1.623^{* * * *}$ & $1.770^{* * * *}$ & $1.417^{* * *}$ \\
\hline City_Den & $1.925^{* * *}$ & $3.080^{* * * *}$ & $4.619^{* * * *}$ & $1.278^{* * * *}$ \\
\hline Country_Exp & $0.214^{* * * *}$ & 0.004 & $0.192^{* * * *}$ & $0.252^{* * * *}$ \\
\hline Country_Den & $2.376^{* * * *}$ & $1.530^{* * * *}$ & $2.876^{* * *}$ & $2.362^{* * *}$ \\
\hline City_PGDP & $-7.280^{* * * *}$ & $-16.240^{* * *}$ & $-8.188^{* * * *}$ & $-6.881^{* * *}$ \\
\hline Dis & $-2.106^{* * *}$ & $-5.287^{* * * *}$ & $-4.670^{* * *}$ & $-0.803^{* * *}$ \\
\hline Country_Pop & $0.162^{* * * *}$ & $0.531^{* * *}$ & $0.627^{* * * *}$ & 0.010 \\
\hline Country_Ins & $0.514^{* * * *}$ & $0.798^{* * *}$ & $3.916^{* * *}$ & $-0.445^{* * *}$ \\
\hline 常数 & $-4.983^{* * * *}$ & $-7.105^{* * *}$ & $-7.504^{* * * *}$ & $-5.523^{* * *}$ \\
\hline$N$ & 15723765 & 15723765 & 15723765 & 15723765 \\
\hline
\end{tabular}

注:***、**、分别表示 $P<0.01 、 P<0.05 、 P<0.1$ 。下同。 
(City-Exp)越丰富。潜在出口企业更有可能将出口 经验中包含的信息转化为出口能力, 做出出口决 策。城市层面的产品技术关联(City-Den)表征了潜 在出口企业出口产品与地方已有出口产品之间在 生产技术、研发创新、消费者市场开拓等方面的知 识重合度。出口企业理性的出口决策以成本最小 和利润最大为目标, 而选择技术关联更高的产品可 以有效降低潜在出口企业的产品信息搜寻成本, 提 升潜在出口企业进人出口市场的概率。

类似地, 目的国出口经验(Country-Exp)溢出也 可以为潜在出口企业学习目的国知识提供便利, 从 而吸引潜在出口企业选择已有出口企业数量较多 的目的国作为出口市场。而且潜在出口企业更倾 向于进人产品技术关联更高的目的国 (CountryDen)。当潜在出口企业所出口产品与目的国已有 出口产品在认知上相互邻近时, 一方面说明目的国 溢出的产品信息与企业需要的产品经验更相近, 企 业更容易通过学习已有产品出口经验降低沉没成 本; 另一方面也说明目的国对类似产品的偏好更 强, 新出口企业将认知邻近的产品出口到对应目的 国市场可以有效规避市场风险。

在此基础上, 观察出口溢出效应对不同所有制 企业的作用差异。除目的国出口经验外,私营企业 对应的核心变量回归系数上均大于其余 2 种所有制 企业。这说明私营企业对出口溢出的依赖程度最 大, 与本文预期相符。国有集体企业在一定程度上 代表政府意志, 因而与地方政府间保有紧密的联 系。外资企业是经济全球化和国际劳动分工的产 物, 因而在对外联系特别是与母国联系方面拥有天 然的优势。与这 2 类企业相比, 私营企业对外联系
和获取信息的能力处于劣势水平, 再加上新出口企 业本身没有出口经验可以遵循, 所以需要通过地 方、全球层面获取更强的知识溢出效益,才能不被 排除在出口市场之外。外资企业对应的目的国出 口经验系数显著大于本土企业, 说明外资企业更倾 向于进人出口企业集聚的目的国。目的国出口企 业数量越多, 知识溢出越强, 但同时企业间竞争也 会越激烈。外资企业的全球化程度更高, 产品技术 和管理经验更先进, 相比于本土企业对国际市场的 适应程度也更好。因而外资新出口企业进人受已 有出口企业挤出效应的负面影响相对更小, 受知识 溢出的正面作用相对更强。

\section{2 跟随型、突破型新企业进入与出口溢出}

在明确出口溢出对新企业进人出口市场作用 的基础上,按新出口企业是否创造新路径而将其划 分为跟随型企业 (未出口新产品且未出口到新目的 国)和突破型企业(出口了新产品或出口到新目的 国), 探究出口溢出对两类企业的作用效果差异, 结 果如表 2 所示。其中模型 5 不区分所有制,模型 6 至 模型 8 区分所有制。

模型 5 中,城市、目的国层面的出口经验、产品 技术关联对应系数均显著为正。这说明有跟随型 企业进入的出口市场中, 出口地、目的国尺度的出 口溢出强度均显著强于突破型企业。跟随型企业 进人出口市场的特征表现为遵循已有出口路径。 因此, 对于潜在出口企业来说, 只有当城市内关于 已有出口产品和出口目的国的相关知识经验较为 丰富时,选择出口已有产品到已有目的国才能够显 著降低学习成本和出口风险,继而有利于制定出 “跟随型”的出口决策。模型 6 至模型 8 中, 城市出

表 2 跟随型、突破型企业进入与出口溢出

Tab.2 Breakthrough and follower firms' entry and export spillover

\begin{tabular}{|c|c|c|c|c|}
\hline \multirow{2}{*}{ 变量 } & 模型 5 & 模型6 & 模型7 & 模型 8 \\
\hline & 不区分所有制 & 国有集体 & 私营 & 外资 \\
\hline City_Exp & $3.851^{* * * *}$ & $5.925^{* * *}$ & $4.487^{* * *}$ & $4.312^{* * * *}$ \\
\hline City_Den & $11.320^{* * * *}$ & $16.240^{* * * *}$ & $19.150^{* * *}$ & $11.440^{* * *}$ \\
\hline Country_Exp & $0.683^{* * *}$ & $1.087^{* * *}$ & $0.755^{* * *}$ & $0.736^{* * *}$ \\
\hline Country_Den & $4.594^{* * *}$ & $6.773^{* * * *}$ & $7.349^{* * * *}$ & $4.549^{* * * *}$ \\
\hline City_PGDP & $17.010^{* * *}$ & $13.610^{* * * *}$ & $33.950^{* * *}$ & $12.150^{* * * *}$ \\
\hline Dis & $-2.188^{* * *}$ & $-5.015^{* * *}$ & $-1.277^{* * *}$ & $-3.127^{* * *}$ \\
\hline Country_Pop & -0.018 & $0.465^{* * *}$ & $0.502^{* * *}$ & -0.040 \\
\hline Country_Ins & $2.896^{* * *}$ & $5.199^{* * * *}$ & $5.370^{* * *}$ & $2.750^{* * * *}$ \\
\hline 常数 & $-6.201^{* * *}$ & $-8.189^{* * *}$ & $-8.308^{* * *}$ & $-6.007^{* * *}$ \\
\hline$N$ & 896222 & 75795 & 283355 & 617915 \\
\hline
\end{tabular}


口经验、产品技术关联系数也均显著为正。这进一 步验证了模型 5 的结果, 即城市、目的国层面出口集 聚溢出效应越强, 跟随型新出口企业进人出口市场 的可能性越大。

观察出口溢出对不同所有制跟随型、突破型企 业的影响, 发现所有制的不同, 会导致2 类新出口企 业对出口溢出的需求差异进一步分异。出口溢出 对本土企业作出跟随型出口决策的促进作用更 强。如前文所述, 本土企业相比于外资企业来说, 出口经验和出口能力更为薄弱, 因而本土潜在出口 企业更需要学习出口地、目的国的出口经验和产品 信息来降低沉没成本和市场风险。跟随型企业对 应着区域已有出口产品和已有目的国。因此相比 于突破型企业来说, 本土跟随型企业更依赖区域和 目的国的已有出口经验, 实现对外出口。

本土企业内部, 国有集体跟随型企业更强调整 体层面的出口地和目的国经验益出。也就是说, 国 有集体跟随型企业更可能出现在不同类型出口企 业集聚的出口地-目的国市场中。而不同于国有集 体企业, 私营跟随型企业进人出口市场更依赖城市 和目的国层面的产品知识溢出, 对应的产品技术关 联系数大于国有集体企业。私营企业受成本最低 和利润最高驱动更明显, 其出口决策更看重地方生 产禀赋和成本优势。城市产品技术关联可以反映 该地区保有的生产禀赋和知识基础, 城市产品技术 关联越高, 说明在该地区生产并出口该产品的成本 越低, 对私营新出口企业的吸引力越大。目的国产 品技术关联可以反映目的国同类产品出口市场的
发育程度, 目的国产品关联越高, 则潜在出口企业 计划出口的产品与目的国出口产品市场邻近度越 高, 向该目的国出口需要克服的沉没成本越低, 同 样有利于私营跟随型企业进人。

\section{3 新企业是否突破到新目的国与出口溢出}

本文进一步分析了出口溢出效应对突破型新 出口企业创造贸易路径类型的影响。根据“是否出 口到所在城市未曾出口过的新目的国”这一标准, 将突破型企业划分为 2 类。以突破型企业进人的城 市一目的国一产品维度数据为样本, 以是否有突破 到新目的国的出口企业进人为被解释变量进行回 归, 探究对于不同类型突破型企业来说, 出口溢出 效应发挥了怎样的作用, 回归结果如表 3 所示。其 中, 模型 9 不区分所有制, 模型 10 至模型 12 识别了 是否有所有制类型为国有集体、私营和外资的突破 型企业向新目的国出口。

首先, 不区分所有制, 分析出口溢出对 2 类突破 型企业进人出口市场的影响。模型 9 中, 城市层面 出口溢出效应的回归系数显著为正, 说明与拓展到 新产品相比,城市层面的出口集聚溢出对新出口企 业拓展到新目的国更有利。分析原因可能在于, 新 目的国之前与出口地没有过贸易联系, 因而目的国 知识溢出也相对较弱。这导致突破到新目的国的 潜在出口企业利用集群对外联系获取出口知识的 难度更大, 从而相比于其他类型新出口企业来说存 在一定劣势。为弥补这一劣势, 城市内部出口集聚 溢出效应需要为潜在出口企业提供更多出口信息, 帮助其克服外部知识匮乏引发的出口成本上升,才

表 3 新出口企业是否突破到新目的国与出口溢出

Tab.3 New export destination entry of new firms and export spillover

\begin{tabular}{|c|c|c|c|c|}
\hline \multirow{2}{*}{ 变量 } & 模型 9 & 模型 10 & 模型 11 & 模型 12 \\
\hline & 不区分所有制 & 国有集体 & 私营 & 外资 \\
\hline City_Exp & $7.554^{* * * *}$ & $4.686^{* * *}$ & 0.232 & $9.621^{* * * *}$ \\
\hline City_Den & $10.040^{* * *}$ & $17.120^{* * *}$ & $17.900^{* * *}$ & $8.332^{* * *}$ \\
\hline Country_Exp & $-0.126^{* * *}$ & $-0.172^{* * *}$ & $-0.378^{* * *}$ & $-0.0695^{* *}$ \\
\hline Country_Den & $-1.230^{* * *}$ & $-0.552^{* * *}$ & $-1.488^{* * *}$ & $-1.308^{* * *}$ \\
\hline City_PGDP & $7.371^{* * * *}$ & 2.145 & $36.570^{* * *}$ & $5.109^{* * * *}$ \\
\hline Dis & $4.981^{* * *}$ & $6.895^{* * * *}$ & $6.230^{* * *}$ & $4.126^{* * * *}$ \\
\hline Country_Pop & $-0.277^{* * *}$ & 0.010 & -0.0429 & $-0.327^{* * *}$ \\
\hline Country_Ins & $-0.260^{* * *}$ & $0.579^{* *}$ & $0.687^{* * *}$ & $-0.369^{* * *}$ \\
\hline 常数 & $-0.084^{* * *}$ & $-1.292^{* * *}$ & $-0.865^{* * *}$ & $0.116^{* * *}$ \\
\hline$N$ & 322976 & 29827 & 65257 & 230837 \\
\hline
\end{tabular}


能保证突破到新目的国的潜在出口企业顺利进入 出口市场。

与城市出口溢出效应相反, 目的国层面出口溢 出效应的回归系数均显著为负, 说明目的国出口溢 出效应会提升突破型企业选择出口到新产品的概 率。原因在于, 出口到新产品的企业对应的出口市 场是已有目的国, 因而能够获取到比目的国突破型 企业更多、更有针对性的出口知识。这些知识对企 业突破到新产品的促进作用要明显高于突破到新 目的国, 并且新目的国与城市之间没有既往贸易联 系, 外部知识向城市内部溢出的途径和效果不如已 有目的国。因此, 突破到新目的国的出口企业无法 从目的国获取到与突破到新产品企业具备同等数 量和质量的出口经验。

其次, 在模型 10 至模型 12 中, 分所有制对 2 种 突破型企业受出口溢出的影响进行分析, 发现国有 集体、私营和外资企业出口路径突破方向受出口溢 出的影响方向与总体回归结果基本相同。除城市 出口经验外, 私营企业对应的回归系数绝对值均大 于国有集体企业和外资企业。这说明, 无论是突破 到新目的国, 还是突破到新产品, 私营突破型企业 更需要城市与目的国层面的出口溢出效应提供必 要的知识和信息。与前文分析类似,国有集体企业 和外资企业可以凭借政府、母国联系获取出口路径 突破必要的知识。相比之下, 私营企业只能依靠全 球、地方层面的知识溢出, 在既有出口经验的基础 上, 创新出口路径, 向新产品和新目的国突破, 所以 对应的回归系数最大。此外, 目的国突破型本土企
业更倾向于选择与城市已有出口产品空间邻近性 更高的产品,产品突破型本土企业更倾向于选择出 口经验更丰富的目的国作为目标市场, 以便弥补在 突破方向上知识贯乏引发的沉没成本上升。

\section{4 稳健性检验}

一些出口企业在研究期内可能出现间断出口 的情况。为了更大程度上避免这类企业在研究中 被识别为新出口企业, 本文对新出口企业进行了更 严格的定义, 即特定编码出口企业只有在 $t-2$ 年至 $t$ 年间均不存在于数据库中, 其 $(t+1)$ 年出现, 才将该 企业识别为 $t$ 年进人出口市场的新企业。据此重新 计算得到的主要实证结果 ${ }^{3}$ 如表 4 中模型 13 至模型 15 所示。另一方面, 中国的直辖市不仅在行政方面 与一般地级市有诸多不同, 而且在经济、产业、人口 等方面都明显超过一般地级市, 为了避免直辖市的 存在给回归结果带来的极端值影响, 本文剔除了样 本中北京、天津、上海和重庆等 4 个直辖市的所有样 本, 进行稳健性检验, 如表 4 中模型 16 至模型 18 所 示。可以发现 2 类稳健性检验结果中主要变量的符 号方向和显著性水平与此前的回归并无明显差别, 具体结果不再赘述。这表明依据本文回归结果得 到的结论具有一定稳健性。

\section{5 结论与讨论}

出口贸易是驱动中国经济增长、重塑中国经济 地理格局的重要力量。出口增长在微观层面体现 为出口企业进人、增长、衰退、退出等空间动态。其

表 4 稳健性检验结果

Tab.4 Results of the robustness tests

\begin{tabular}{|c|c|c|c|c|c|c|}
\hline \multirow{3}{*}{ 变量 } & \multicolumn{3}{|c|}{ 新定义新出口企业 } & \multicolumn{3}{|c|}{ 剔除直辖市样本 } \\
\hline & 模型 13 & 模型 14 & 模型 15 & 模型 16 & 模型 17 & 模型 18 \\
\hline & 基本回归 & 突破/跟随 & 新目的国 & 基本回归 & 突破/跟随 & 新目的国 \\
\hline City_Exp & $0.814^{* * * *}$ & $2.679^{* * *}$ & $11.360^{* * * *}$ & $1.044^{* * * *}$ & $4.981^{* * * * *}$ & $6.038^{* * * *}$ \\
\hline City_Den & $2.102^{* * * *}$ & $6.334^{* * *}$ & $8.630^{* * * *}$ & $1.552^{* * * *}$ & $7.615^{* * * *}$ & $13.870^{* * *}$ \\
\hline Country_Exp & $0.273^{* * * *}$ & $1.457^{* * * *}$ & $-0.126^{* * *}$ & $0.388^{* * * *}$ & $0.511^{* * * *}$ & $-0.176^{* * *}$ \\
\hline Country_Den & $3.589^{* * * *}$ & $5.099^{* * *}$ & $-1.114^{* * *}$ & $1.958^{* * * *}$ & $4.122^{* * * * *}$ & $-1.701^{* * *}$ \\
\hline City_PGDP & $-9.281^{* * *}$ & $24.000^{* * *}$ & $26.800^{* *}$ & $-7.430^{* * *}$ & $22.810^{* * * *}$ & $5.338^{* * * *}$ \\
\hline Dis & $-0.782^{* * *}$ & -1.271 & 1.503 & $-1.882^{* * * *}$ & $-0.918^{*}$ & $3.760^{* * * *}$ \\
\hline Country_Pop & $0.463^{* * * *}$ & -0.497 & -11.211 & $0.256^{* * *}$ & 0.102 & $-0.719^{* * *}$ \\
\hline Country_Ins & $0.964^{* * *}$ & $3.984^{* * *}$ & $-3.235^{*}$ & $0.403^{* * * *}$ & $1.792^{* * * *}$ & $-0.468^{* * * *}$ \\
\hline$N$ & 6806828 & 366976 & 192080 & 13349970 & 746325 & 284409 \\
\hline
\end{tabular}

(3) 主要回归结果指表 1 至表 3 中不区分所有制的 3 个主回归,其中, 模型 13 对应模型 1 , 模型 14 对应模型 5 , 模型 15 对应模型 9 。 
中,新出口企业进人至关重要。国际贸易理论指出 集聚引发的知识溢出是影响出口行为空间区位的 关键因素,而且企业异质性的存在使不同类型出口 企业面临不同的沉没成本和市场进人门槛。出口 溢出包含地方和全球 2 个维度。地方层面的出口溢 出有利于邻近的出口企业通过交流学习降低沉没 成本; 全球层面的出口溢出借助区域对外贸易联 系, 将外部知识引人区域内部, 为出口企业所用。 演化经济地理理论认为认知邻近是出口企业获取 到有效知识溢出的重要前提。出口地、目的国维度 的企业出口经验、产品技术关联是出口企业学习的 关键内容。

本文利用2002-2011 年中国海关贸易数据库, 识别中国对外出口市场的新企业进人, 并从地方、 全球 2 个维度出发, 探究出口溢出对新企业进人出 口市场的作用, 主要结论如下:

(1) 从出口地维度看, 新出口企业进人率由内 陆向沿海递减。跟随型企业集中于东部沿海, 突破 型企业集中于中西部地区。从目的国维度看, 新企 业进人率较高的经济体中, 部分与中国在地理上邻 近, 另一部分与中国有紧密的贸易联系。跟随型企 业集中分布于中国的主要贸易伙伴国家; 突破型企 业则相反。

(2) 出口地、目的国维度出口溢出可以显著促 进新出口企业进人, 私营企业对出口溢出的需求程 度最高, 外资企业更可能出现在出口企业集聚的目 的国。

(3) 在新企业内部, 出口溢出对跟随型企业, 特 别是本土跟随型企业进人的促进作用更强。本土 企业中, 国有集体企业更强调整体出口经验溢出, 私营企业更强调产品知识溢出。

(4) 城市层面的出口溢出更有利于新出口企业 拓展到新目的国, 目的国层面出口溢出更有利于新 出口企业拓展到新产品。私营企业更需要凭借出 口溢出实现出口路径突破。

本文在考虑出口地知识溢出的同时, 增加目的 国维度出口溢出, 同时识别企业异质性的影响, 有 助于明确出口企业动态差异及其深层机制。此外, 本文研究也有一定政策意义。首先, 城市需要从地 方内部和对外联系 2 个层面人手, 改善贸易环境, 提 升新企业进人出口市场的可能性。其次, 私营企业 是推动中国出口市场发展的关键力量, 地方政府应
注重培育本地市场活力,激发知识交换和学习模 仿, 以充分发挥私营企业对出口贸易增长的带动作 用。最后, 突破型企业是推动城市出口产品和出口 目的国多样化的微观主体。欲拓展出口市场的扩 展边际, 区域更需要加强企业集聚和产品多样化发 展,激发企业创新活力, 实现出口路径的创造。

\section{参考文献(References)}

郭琪. 2016. 中国制造业出口多样化及其空间动态演化研究

[D]. 北京: 北京大学. [Guo Q. 2016. Export diversification and its spatial evolution in Chinese manufacturing sectors. Beijing, China: Peking University. ]

贺灿飞, 董瑶, 周沂. 2016. 中国对外贸易产品空间路径演化 [J]. 地理学报, 71(6): 970-983. [He C, Dong Y, Zhou Y. 2016. Evolution of export product space in China: Path-dependent or path-breaking? Acta Geographica Sinica, 71(6): 970-983. ]

韦伯. 1997. 工业区位论 [M]. 李刚剑, 等, 译. 北京: 商务印书 馆. [Weber A. et al. 1997. Theory of the location of industries. Tranlated by Li G J, et al. Beijing, China: The Commercial Press. ]

Ahn J, Khandelwal A K, Wei S J. 2011. The role of intermediaries in facilitating trade $[\mathrm{J}]$. Journal of International Economics, 84(1): 73-85.

Aitken B J, Hanson G H, Harrison A E. 1997. Spillovers, foreign investment, and export behavior [J]. Journal of International Economics, 43(1): 103-132.

Baldwin R E, Rieder R. 2007. A test of endogenous trade bloc formation theory on EU data $[\mathrm{J}]$. Social Science Electronic Publishing, 11(2): 77-112.

Bathelt H, Malmberg A, Maskell P. 2004. Clusters and knowledge: Local buzz, global pipelines and the process of knowledge creation [J]. Progress in Human Geography, 28 (1): $31-56$

Bathelt H, Schuldt N. 2010. International trade fairs and global buzz, part I: Ecology of global buzz [J]. European Planning Studies, 18(12): 1957-1974.

Bernard A B, Jensen J B. 2004. Entry, expansion, and intensity in the US export boom, 1987-1992 [J]. Review of International Economics, 12(4): 662-675.

Boschma R A. 2005. Proximity and innovation: A critical assessment [J]. Regional Studies, 39: 61-74.

Boschma R A, Iammarino S. 2009. Related variety, trade linkages, and regional growth in Italy [J]. Economic Geography, 85(3): 289-311. 
Boschma R A, Minondo A, Navarro M. 2012. Related variety and regional growth in Spain [J]. Papers in Regional Science, 91(2): 241-256.

Boschma R A, Wenting R. 2007. The spatial evolution of the British automobile industry: Does location matter? [J]. Industrial and Corporate Change, 16(2): 213-238.

Chaney T. 2014. The network structure of international trade [J]. American Economic Review, 104(11): 3600-3634.

Dosi G. 1997. Opportunities, incentives and the collective patterns of technological change $[\mathrm{J}]$. The Economic Journal, 107: 1530-1547.

Gertler M S. 1995. "Being there": proximity, organization, and culture in the development and adoption of advanced manufacturing technologies [J]. Economic Geography, 71(1): 126.

Grabher G. 2002. Cool projects, boring institutions: Temporary collaboration in social context $[\mathrm{J}]$. Regional Studies, 36(3): 205-214.

Hidalgo C A, Klinger B, Barabasi A L, et al. 2007. The product space and its consequences for economic growth $[\mathrm{J}]$. Science, 317: 482-487.

Jacobs J. 1969. The economy of cities [M]. New York, NY: Random House.

Koenig P. 2009. Agglomeration and the export decisions of French firms [J]. Journal of Urban Economics, 66(3): 186-195.

Koenig P, Mayneris F, Poncet S. 2010. Local export spillovers in France [J]. European Economic Review, 54(4): 622-641.

Krugman P. 1979. Increasing returns, monopolistic competition and international trade $[\mathrm{J}]$. Journal of International Economics, 9(4): 469-479.

Malecki E J. 2010. Global knowledge and creativity: New challenges for firms and regions [J]. Regional Studies, 44 (8): 1033-1052.

Malmberg A, Lundequist P. 2000. Agglomeration and firm per- formance: Economies of scale, localisation, and urbanisation among Swedish export firms $[\mathrm{J}]$. Environment and Planning A, 32(2): 305-322.

Manova K, Zhang Z. 2012. Export prices across firms and destinations $[\mathrm{J}]$. The Quarterly Journal of Economics, 127(1): 379-436.

Marshall A. 1920. Principles of economics [M]. London, UK: Macmillan.

Martin R. 2010. Roepke lecture in economic geography-rethinking regional path dependence: Beyond lock-in to evolution [J]. Economic Geography, 86: 1-27.

Maskell P, Malmberg A. 2007. Myopia, knowledge development and cluster evolution [J]. Journal of Economic Geography, 7(5): 603-618.

Melitz M J. 2003. The impact of trade on intra-industry reallocations and aggregate industry productivity $[\mathrm{J}]$. Econometrica, 71(6): 1695-1725.

Nooteboom B. 2001. Learning and innovation in organizations and economies [M]. Oxford, UK: Oxford University Press.

Ottaviano G, Tabuchi T, Thisse J F. 2002. Agglomeration and trade revisited $[\mathrm{J}]$. International Economic Review, 43(2): 409-435.

Poncet S. 2013. Export upgrading and growth: The prerequisite of domestic embeddedness [J]. World Development, 51 (5): 104-118.

Rinallo D, Borghini S, Golfetto F. 2010. Exploring visitor experiences at trade shows [J]. Journal of Business \& Industrial Marketing, 25(4): 249-258.

Timmermans B, Boschma R. 2014. The effect of intra-and inter-regional labour mobility on plant performance in Denmark: The significance of related labour inflows $[\mathrm{J}]$. Journal of Economic Geography, 14(2): 289-311.

Torre A. 2008. On the role played by temporary geographical proximity in knowledge transmission $[\mathrm{J}]$. Regional Studies, 42(6): 869-889. 


\title{
Impact of export spillovers on the entry of new firms into the export market
}

\author{
HE Canfei ${ }^{1,2}$, HU Xuqian ${ }^{1,2}$, LUO Qian ${ }^{1,2}$ \\ (1. School of Urban and Environmental Sciences, Peking University, Beijing 100871, China; \\ 2. Peking University-Lincoln Institute Center for Urban Development and Land Policy, Beijing 100871, China)
}

\begin{abstract}
Export growth is a key driver for China's economic development. The entry of new export firms is an important indicator of export growth. The New Trade Theory believes that knowledge spillover brought about by agglomeration externalities is a crucial factor affecting the exporting decision making of firms. Global and local knowledge spillovers encourage firms to acquire necessary export knowledge and thus reduce their difficulty of entering export markets. Theories of evolutionary economic geography emphasize that cognitive proximity is the prerequisite for the validity of export spillovers. Global and local export experience and product proximity are the main contents of export spillovers. This study used China Customs Trade Data from 2002 to 2011 to analyze the impact of export spillovers on the entry of new firms into the export market. The results show that export spillovers can significantly increase the probability of new exporters entering the export market. Private firms are more likely to enter markets with stronger spillovers. Besides, export spillovers have a stronger role in promoting the entry of follower firms, especially domestic firms. State-owned collective enterprises emphasize the overflow of overall export experience, and private companies place more emphasis on product knowledge spillovers. Local spillovers are beneficial for the expansion of new exporting firms to new destination countries, while global spillovers are useful for new exporters expanding into new product areas. This study improved the research on the spatial dynamics of export firms, expanded the spatial dimension in which export spillovers affect export decisions, and helped deepen the understanding of China's trade market.
\end{abstract}

Keywords: export spillovers; global-local interaction; entry of new export firms; China 